DESY-11-262

IFJPAN-IV-2011-15

OUTP-11-38-P

\title{
Forward Jets and Energy Flow in Hadronic Collisions
}

\author{
M. Deak, ${ }^{1,2}$ F. Hautmann, ${ }^{3}$ H. Jung, ${ }^{4,5,6}$ and K. Kutak ${ }^{7}$ \\ ${ }^{1}$ IFT-UAM/CSIC, Universidad Autónoma de Madrid, E-28049 Madrid \\ ${ }^{2}$ Departamento de Física de Partículas, \\ Universidade de Santiago de Compostela, E-15782 Santiago de Compostela \\ ${ }^{3}$ Theoretical Physics Department, University of Oxford, Oxford OX1 3NP \\ ${ }^{4}$ Deutsches Elektronen Synchrotron, D-22603 Hamburg \\ ${ }^{5}$ CERN, Physics Department, CH-1211 Geneva 23 \\ ${ }^{6}$ Elementaire Deeltjes Fysica, Universiteit Antwerpen, B 2020 Antwerpen \\ ${ }^{7}$ Instytut Fizyki Jadrowej im H. Niewodniczanskiego, PL 31-342 Krakow
}

\begin{abstract}
We observe that at the Large Hadron Collider, using forward + central detectors, it becomes possible for the first time to carry out calorimetric measurements of the transverse energy flow due to "minijets" accompanying production of two jets separated by a large rapidity interval. We present parton-shower calculations of energy flow observables in a high-energy factorized Monte Carlo framework, designed to take into account QCD logarithmic corrections both in the large rapidity interval and in the hard transverse momentum. Considering events with a forward and a central jet, we examine the energy flow in the interjet region and in the region away from the jets. We discuss the role of these observables to analyze multiple parton collision effects.
\end{abstract}


The production of final states created with high momentum transfers and boosted to forward rapidities is a new feature of the Large Hadron Collider compared to previous collider experiments, subject of intense experimental and theoretical activity [1]. Forward high- $\mathrm{p}_{\perp}$ production enters the LHC physics program in both new particle discovery processes (e.g., jet studies in decays of boosted massive states [2]) and new aspects of standard model physics (e.g., QCD at small $x$ and its interplay with cosmic ray physics [3] ]).

Investigating such final states poses new challenges to both experiment and theory. On one hand, measurements of jet observables in the forward region call for new experimental tools and analysis techniques [1, 4, 5]. On the other hand, the evaluation of QCD theoretical predictions is made complex by the forward kinematics forcing high- $\mathrm{p}_{\perp}$ production into a region characterized by multiple hard scales, possibly widely disparate from each other. This raises the issue of whether potentially large corrections arise beyond finite-order perturbation theory which call for perturbative QCD resummations [6 8 ] and/or contributions beyond single parton interaction [9 13]. It is thus relevant to ask to what extent current Monte Carlo generators can provide realistic event simulations of forward particle production, and how LHC experimental measurements can help improve our understanding of QCD effects in the forward region.

To this end, in [8, 14] we have proposed measuring correlations of a forward and a central jet and performed a numerical analysis of the effects of noncollinear, high-energy corrections to initial-state QCD showers. First experimental studies have since appeared in preliminary form [15]. The predictions [14] for the forward jet spectra are in reasonable agreement with the data.

In this paper we point out that the capabilities of forward + central detectors at the LHC allow one to perform more detailed studies of the event structure, by measuring the associated transverse energy flow as a function of rapidity, both in the interjet region and in the region away from the trigger dijets. Such energy flow measurements have not been made before at hadron-hadron colliders. We observe that as a result of the large phase space opening up for high- $\mathrm{p}_{\perp}$ production at LHC center-of-mass energies, one has an average transverse energy flow per unit rapidity of $10 \mathrm{GeV}$ or more out to forward rapidity. Then it becomes possible to carry out measurements of the flow resulting from "mini-jets" with transverse energy above a few $\mathrm{GeV}$, thus suppressing the sensitivity of the observable to soft particle production. We suggest this minijet energy flow as a way to investigate the detailed structure of events with forward and central jets.

These measurements can be viewed as complementary to measurements performed by the CMS Collaboration [16] on the energy flow in the forward direction in minimum bias events and in events containing a central dijet system. The studies [16] are designed to investigate properties of the soft underlying event; in particular, they illustrate that the energy flow observed in the forward region is not well described by tunes of the PYTHIA Monte Carlo generator [11, 12] based on charged particle spectra in the central region, especially for the minimum bias sample. The energy flow measurements discussed in this paper, on the other hand, can serve to investigate features of events that depend on (semi)hard color radiation. For proposed studies of forward event shapes and correlations to investigate minimum bias, see [17].

Note that the analysis discussed in this paper can be extended to the case of forwardbackward jets. Here one can look for Mueller-Navelet effects [1, 4, [6]. Investigating QCD radiation associated with forward-backward jets will serve to analyze backgrounds in Higgs searches from vector boson fusion channels [18] and studies based on a central jet veto [19] 
to extract information on Higgs couplings [20]. In this case too the underlying jet activity accompanying the Higgs may receive comparable contributions 21] from finite-angle radiative contributions to single-chain showers, extending across the whole rapidity range, and from multiple-parton interactions.

Our focus in this work is on initial-state effects in energy flow measurements. Finalstate effects such as those discussed in [22, 23] may require resummation of logarithmically enhanced terms arising from restrictions on the phase space in which color radiation is considered and depending on the algorithms used to reconstruct the jets. We do not address these potential effects in this paper. See also [24 26] for recent analyses of energy flow observables.

We next discuss the energy flow according to the method [8, 14] and then present numerical results.

Let us start by considering final states associated with the production of a forward and a central jet at the LHC as proposed in [14] (Fig. 11). To be specific, we take

$$
1<\eta_{c}<2, \quad-5<\eta_{f}<-4
$$

where $\eta_{c}$ and $\eta_{f}$ are the central and forward jet pseudorapidities. We consider the transverse energy flow as a function of pseudorapidity

$$
\frac{d E_{\perp}}{d \eta}=\frac{1}{\sigma} \int d q_{\perp} q_{\perp} \frac{d \sigma}{d q_{\perp} d \eta}
$$

The energy flow is sensitive to color radiation associated with the trigger specified in Eq. (10), and its understanding requires treating multiple QCD emission by parton showering or resummation methods beyond finite perturbative order.

Observe that the transverse factor $q_{\perp}$ in the integrand on the right hand side in Eq. (2) enhances the sensitivity to the high momentum transfer end of the QCD parton cascades compared to the inclusive jet cross sections. On one hand, it makes the transverse momentum ordering approximation less physically justified in the long-time evolution of the parton cascade. On the other hand, it increases the importance of corrections due to extra hardparton emission in the jet production subprocess at the shortest time scales.

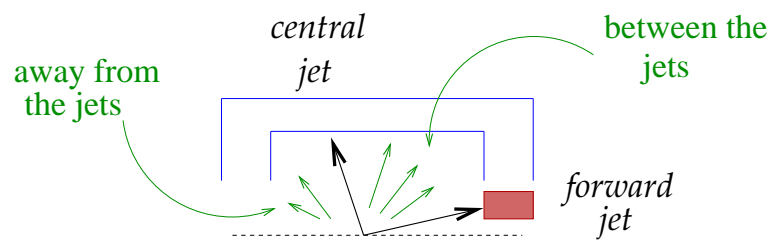

FIG. 1. Production of forward and central jets: energy flow in the inter-jet and outside regions.

For these reasons, it is physically well-motivated to analyze the energy flow by employing the approach suggested in [14], in which one couples the short distance forward-jet matrix elements [8], which contain extra hard-gluon emission via high-energy factorization [7], to the transverse-momentum dependent parton showers [27, 28], which go beyond the collinear ordering approximation by Monte Carlo implementation of CCFM evolution 11 In this frame-

\footnotetext{
${ }^{1}$ A study of the phenomenological consequences of the dynamical effects at finite $\mathrm{k}_{\perp}$, in both matrix elements and parton showers, on final states with multiple jets may be found in [29].
} 
work we write the cross section in Eq. (2) as

$$
\frac{d \sigma}{d q_{\perp} d \eta}=\sum_{a} \int \phi_{a / A} \otimes \frac{d \widehat{\sigma}}{d q_{\perp} d \eta} \otimes \phi_{g^{*} / B}
$$

where $\otimes$ specifies a convolution in both longitudinal and transverse momenta, $\widehat{\sigma}$ is the hard scattering cross section [8], while $\phi_{a / A}$ and $\phi_{g^{*} / B}$ are respectively the collinearly factorized and high-energy factorized initial-state distributions, obtained as in [14] from, respectively, the on-shell and off-shell backward shower evolution of the two highly asymmetric incoming parton states that initiate the hard scatter 2 The approach [8, 14] is designed to take into account QCD logarithmic corrections both in the large rapidity interval and in the hard transverse momentum.

Besides the effects of emission of high transverse momenta, the energy flow (2) receives contributions from emission of soft particles. The calculational framework described above includes soft parton emission through the $1 /(1-z)$ terms in the branching vertices and the form factors in the shower evolution [27], which contain both Sudakov and "non-Sudakov" contributions. We here point out that, unlike previous collider experiments, at the LHC it is possible to reduce the infrared sensitivity of energy flow measurements by exploiting the large phase space available for production of high $\mathrm{p}_{\perp}$, as follows.

Note that previous measurements of transverse energy flow were made in lepton-proton collisions at HERA [30], where one had roughly an average transverse energy of $1 \div 2 \mathrm{GeV}$ per unit rapidity. This increases by a factor of five at the LHC [16] to about $5 \div 10 \mathrm{GeV}$ or more per unit rapidity, as a result of the phase space opening up for high- $\mathrm{p}_{\perp}$ production. Besides the standard energy flow in Eq. (2) obtained by summing the energies over all particles in the final states, we will thus consider also a scenario in which first we merge particles into jets by means of a jet algorithm, and then we construct the associated energy flow from jets with transverse energy above a given lower bound $q_{0}$,

$$
q_{\perp}>q_{0}, \quad q_{0}=\mathcal{O}(\text { a few } \mathrm{GeV}) .
$$

The infrared safety is ensured by the use of the jet algorithm to cluster particles. On the other hand, the use of a lower bound $q_{0}$ on the order of a few $\mathrm{GeV}$ is made possible by the large transverse energy per unit rapidity at the LHC. In the calculations that follow we take $q_{0}=5 \mathrm{GeV}$ in Eq. (4). We call this the associated "mini-jet" energy flow.

In addition to radiative corrections from multiple emission in a single parton collision, the evaluation of $d E_{\perp} / d \eta$ is sensitive to contributions of multiple parton collisions [10]. In the calculations that follow we will estimate these contributions using the Monte Carlo model [12].

In the following we consider events with dijets (Fig. 1) reconstructed using the Siscone algorithm [31] with $R=0.4$, in the rapidity region (11). We will consider the cases of jets with transverse energy $\mathrm{E}_{\perp}>10 \mathrm{GeV}$.

Fig. 2 shows the transverse energy flow in the interjet region for the cases of particle flow and of minijet flow. Besides the calculation above given by the curves labelled CASCADE, we report results obtained from Pythia [12] and Powheg [32] Monte Carlo event generators. Pythia 12] is used in two different modes, with multiple parton interactions (PYTHIA-mpi, tune Z1 [33]) and without multiple parton interactions (PYTHIA-nompi). The particle energy

${ }^{2}$ See Refs. [14, 29] for discussions of the use of the initial-state distributions in Eq. (3). 
flow plot on the left in Fig. 2 shows the jet profile picture, and indicates enhancements of the energy flow in the inter-jet region with respect to the PYTHIA-nompi result from higher order emissions in CASCADE and from multiple parton collisions in PYTHIA-mpi. On the other hand, there is little effect from the next-to-leading hard correction in PowHEG with respect to PYTHIA-nompi. The energy flow is dominated by multiple-radiation, partonshower effects.
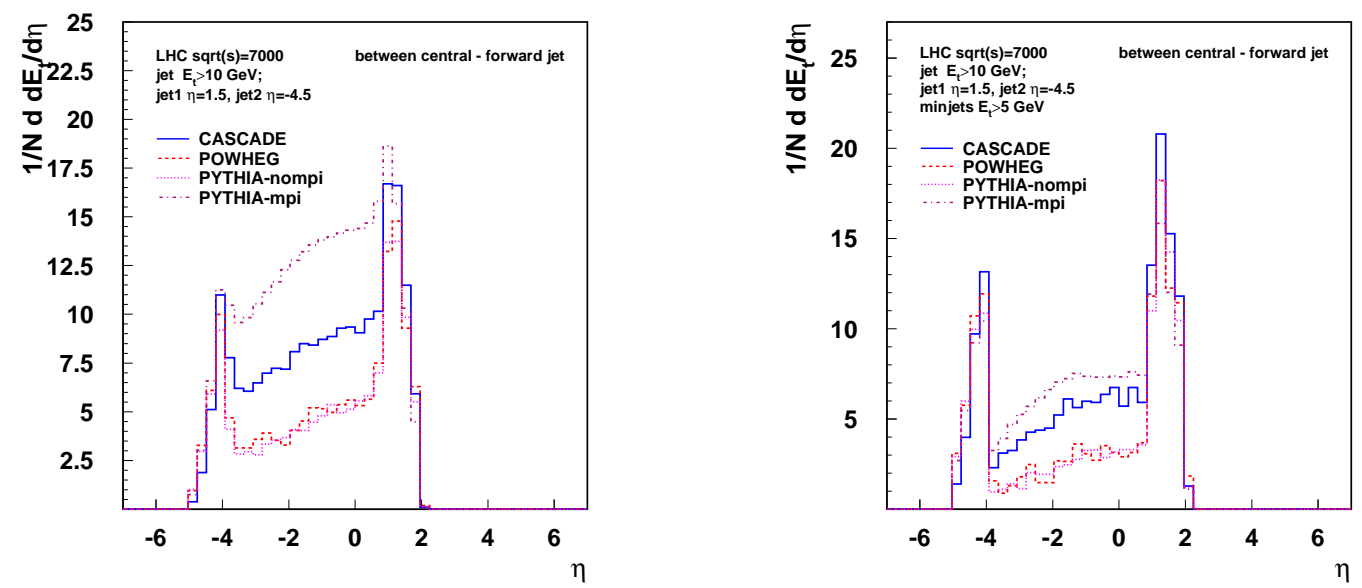

FIG. 2. Transverse energy flow in the inter-jet region: (left) particle flow; (right) minijet flow.

The minijet energy flow plot on the right in Fig. 2 indicates similar effects, with reduced sensitivity to infrared radiation. As the minijet flow definition suppresses the contribution of soft radiation, the CASCADE and PYTHIA-mpi results become more similar in the inter-jet region. Particle and minijet flow measurements could be used to investigate the detailed form of multiparton radiation effects. In particular, these results are of interest for the QCD tuning of Monte Carlo generators, especially in connection with the estimation of QCD backgrounds in search channels involving two jets far apart in rapidity such as Higgs boson searches from vector boson fusion [18, 20].
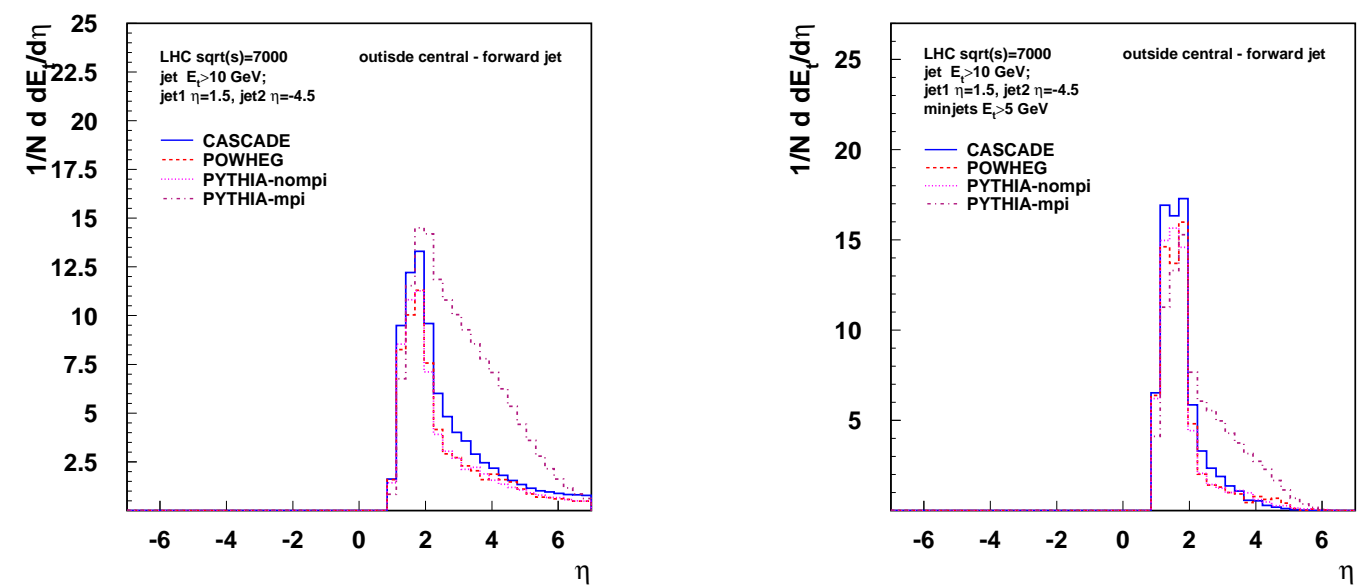

FIG. 3. Transverse energy flow in the outside region: (left) particle flow; (right) minijet flow.

It is also of interest to measure the energy flow in the outside region corresponding to rapidities opposite to the forward jet, far in the backward region. Results for this are shown 
in Fig. 3. In the outside region an enhancement of the energy flow can be produced by showering from multiple parton chains, while single-chain calculations give a suppression of the transverse flow, mainly due to phase space. In this region one is sampling also contributions from the initial-state decay chain at substantially larger values of longitudinal momenta, where the effects of corrections to collinear ordering, taken into account by the CASCADE result, are not large. On the other hand, contributions from multiple showers could be significant, due to gluon radiation shifting to larger values of $x$ in each of the sequential parton chains, as the total energy available to the collision is shared between the different chains.
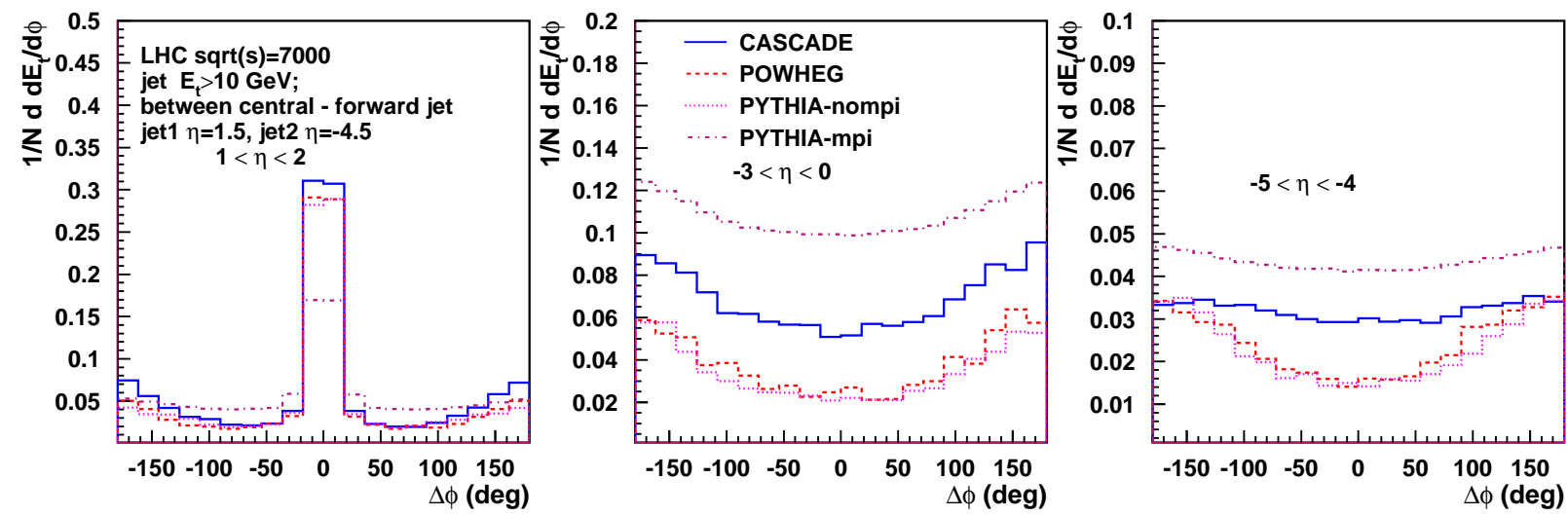

FIG. 4. Azimuthal dependence of the particle energy flow for different rapidity ranges: (left) centraljet; (middle) intermediate; (right) forward-jet.

In Figs. 4 and 5 we examine the azimuthal dependence of the transverse energy flow, respectively for the particle flow and minijet scenarios. Here $\Delta \phi$ is measured with respect to the central jet. The $\Delta \phi$ distribution is shown for three different rapidity ranges, corresponding to the central-jet, forward-jet, and intermediate rapidities.
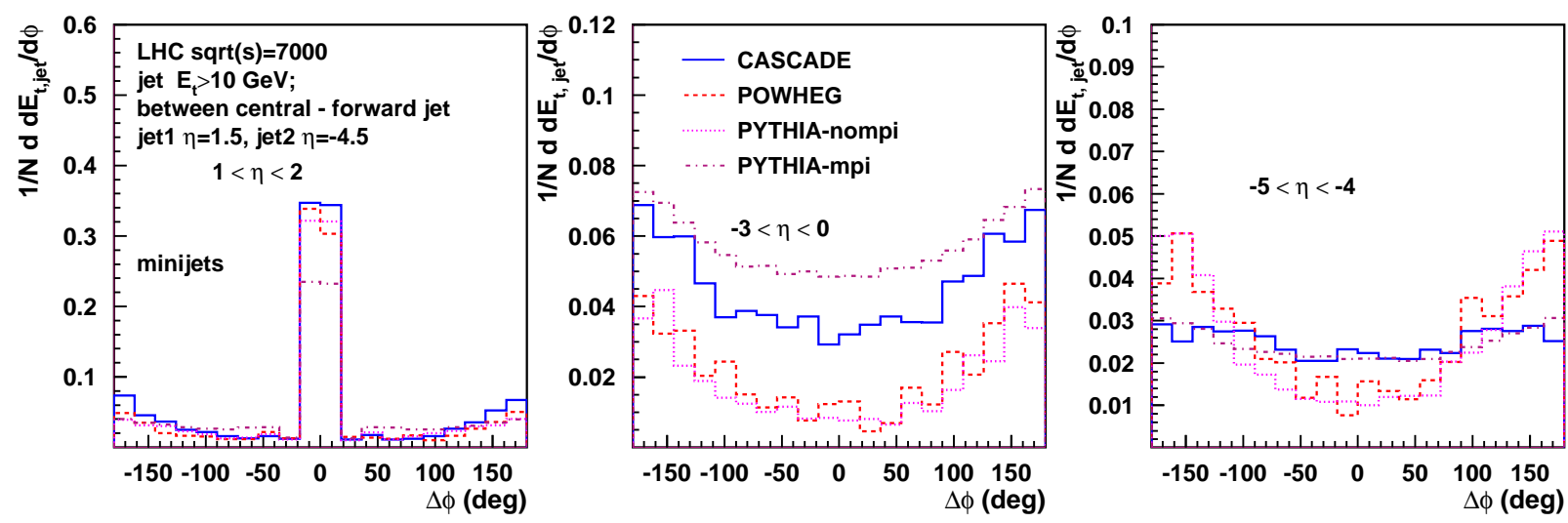

FIG. 5. Azimuthal dependence of the minijet energy flow for different rapidity ranges: (left) centraljet; (middle) intermediate; (right) forward-jet.

We see from Figs. 4 and 5 that, as we go toward forward rapidity, the CASCADE and PyTHIA-mpi calculations give a more pronounced flattening of the $\Delta \phi$ distribution compared to Powheg and PYTHIA-nompi, corresponding to increased decorrelation between the jets. The effect occurs for both the particle flow and the minijet flow. In the minijet case (Fig. 5) 
the sensitivity to soft radiation is reduced. One of the by-products of this is that also the difference between the CASCADE and PYTHIA-mpi results decreases.

Let us summarize. Forward jets observed at the LHC for the first time are roughly in agreement with predictions from different Monte Carlo event generators. In this note we have proposed more exclusive measurements to probe the associated event structure.

We have studied the transverse energy flow associated with the hadro-production of a forward and a central jet. These measurements can be performed with the LHC by exploiting the forward calorimeters; their sensitivity to soft particle production can be reduced by exploiting the large phase space available for high- $\mathrm{p}_{\perp}$ production at LHC center-of-mass energies. The energy flow studies we have considered can be helpful for the QCD tuning of Monte Carlo event generators and for searches of new physics signals based on analyses of jet final states.

We have presented Monte Carlo results that contain the small- $x$, large-rapidity resummation of QCD logarithmically enhanced terms based on the forward-jet matrix elements [8, 14]. This is combined with large- $x$ effects according to the CCFM prescription implemented in the parton shower event generator [27, 28]. We have obtained results for the energy flow in the inter-jet region and in the region away from the jets. We have also estimated the effects of the multiple parton interactions taken into account by [12], and found that measurements of the energy flow in the inter-jet and outside regions can provide distinctive features to analyze such effects.

Phenomenological studies of color radiation activity along the lines suggested in this paper could be applied to examining QCD backgrounds in search channels from vector boson fusion [18, 19]. From the theory viewpoint, energy flow observables such as those considered here are of interest both for studies of initial-state distributions that generalize ordinary parton distributions [34 36] to more exclusive descriptions of event structure and for studies of final-state infrared effects [22 24] associated with emission of color in restricted phase space regions.

Acknowledgments. We thank the CERN Theory Division and LHC Physics Center for hospitality. M. D. and F. H. thank UniverseNet for support. K. K. acknowledges the support of NCBiR grant nr LIDER/02/35/L-2/10/NCBiR/2011.

[1] Z. Ajaltouni et al., arXiv:0903.3861 [hep-ph].

[2] A. Abdessalam et al., arXiv:1012.5412 [hep-ph].

[3] M. Grothe, F. Hautmann and S. Ostapchenko, arXiv:1103.6008 [hep-ph].

[4] D. d'Enterria, arXiv:0911.1273 [hep-ex].

[5] S. Abdullin et al., Eur. Phys. J. C 53 (2008) 139.

[6] A.H. Mueller and H. Navelet, Nucl. Phys. B282 (1987) 727; C. Ewerz et al., J. Phys. G26 (2000) 696; S. Catani et al., Nucl. Phys. B Proc. Suppl. 29A (1992) 182.

[7] S. Catani, M. Ciafaloni and F. Hautmann, Phys. Lett. B242 (1990) 97; Nucl. Phys. B366 (1991) 135; Phys. Lett. B307 (1993) 147; S. Catani and F. Hautmann, Phys. Lett. B315 (1993) 157; Nucl. Phys. B427 (1994) 475.

[8] M. Deak, F. Hautmann, H. Jung and K. Kutak, JHEP 0909 (2009) 121; arXiv:0908.1870.

[9] T. Sjöstrand and M. van Zijl, Phys. Rev. D36 (1987) 2019; Phys. Lett. B188 (1987) 149. 
[10] P. Bartalini and L. Fanò (eds.), arXiv:1003.4220 [hep-ph], Proceedings 1st MPI Workshop (Perugia, 2008), DESY-PROC-2009-06; P. Bartalini et al., arXiv:1111.0469 [hep-ph].

[11] T. Sjöstrand, S. Mrenna, and P. Skands, JHEP 0605 (2006) 026.

[12] P. Skands, Phys. Rev. D82 (2010) 074018.

[13] J.R. Gaunt and W.J. Stirling, JHEP 1106 (2011) 048; JHEP 1003 (2010) 005; B. Blok, Yu. Dokshitzer, L. Frankfurt and M. Strikman, Phys. Rev. D83 (2011) 071501; M. Strikman and W. Vogelsang, Phys. Rev. D83 (2011) 034029; T.C. Rogers and M. Strikman, Phys. Rev. D81 (2010) 016013; G. Calucci and D. Treleani, Phys. Rev. D83 (2011) 016012; Phys. Rev. D80 (2009) 054025; Phys. Rev. D79 (2009) 074013; F.A. Ceccopieri, Phys. Lett. B697 (2011) 482; M. Diehl and A. Schäfer, Phys. Lett. B698 (2011) 389; M. Diehl, D. Ostermaier and A. Schäfer, arXiv:1111.0910 [hep-ph]; S. Domdey, H.-J. Pirner and U.A. Wiedemann, Eur. Phys. J. C 65 (2010) 153; M.G. Ryskin and A.M. Snigirev, Phys. Rev. D83 (2011) 114047; E. Maina, JHEP 1101 (2011) 061; JHEP 0909 (2009) 081; JHEP 0904 (2009) 098; E.L. Berger, C.B. Jackson and G. Shaughnessy, Phys. Rev. D81 (2010) 014014.

[14] M. Deak, F. Hautmann, H. Jung and K. Kutak, arXiv:1012.6037 [hep-ph]; F. Hautmann, arXiv:1101.2656 [hep-ph]; PoS ICHEP2010 (2010) 108.

[15] CMS Coll., CMS-PAS-FWD-10-003; CMS-PAS-FWD-10-006.

[16] CMS Coll. (S. Chatrchyan et al.), JHEP 1111 (2011) 148.

[17] P. Skands and K. Wraight, Eur. Phys. J. C 71 (2011) 1628.

[18] K.J.C. Leney [on behalf of the ATLAS Coll.], arXiv:0810.3144 [hep-ex]; M. Vazquez Acosta [on behalf of the CMS Coll.], arXiv:0901.3098 [hep-ex].

[19] J.M. Butterworth, B.E. Cox and J.R. Forshaw, Phys. Rev. D65 (2002) 096014.

[20] B.E. Cox, J.R. Forshaw and A.D. Pilkington, Phys. Lett. B696 (2011) 87.

[21] M. Deak et al., arXiv:1006.5401 [hep-ph]; F. Hautmann, H. Jung and V. Pandis, arXiv:1011.6157 [hep-ph]; F. Hautmann, arXiv:0909.1240; Phys. Lett. B 535 (2002) 159.

[22] A. Banfi, M. Dasgupta, K. Khelifa-Kerfa and S. Marzani, JHEP 1008 (2010) 064.

[23] C.F. Berger, T. Kucs and G. Sterman, Phys. Rev. D68 (2003) 014012.

[24] I. Sung, Phys. Rev. D80 (2009) 094020.

[25] Y. Hatta and T. Ueda, Phys. Rev. D80 (2009) 074018.

[26] A. Papaefstathiou, J.M. Smillie and B.R. Webber, JHEP 1004 (2010) 084.

[27] H. Jung et al., Eur. Phys. J. C 70 (2010) 1237.

[28] H. Jung, Comput. Phys. Commun. 143 (2002) 100.

[29] F. Hautmann and H. Jung, JHEP 0810 (2008) 113; arXiv:0804.1746; arXiv:0805.4786.

[30] C. Adloff et al. [H1 Coll.], Eur. Phys. J. C 12 (2000) 595.

[31] M. Cacciari and G.P. Salam, Phys. Lett. B 641 (2006) 57; M. Cacciari, G.P. Salam and G. Soyez, http://fastjet.fr; G.P. Salam and G. Soyez, JHEP 0705 (2007) 086.

[32] S. Alioli, P. Nason, C. Oleari and E. Re, JHEP 1104 (2011) 081.

[33] R.D. Field, arXiv:1010.3558 [hep-ph].

[34] F. Hautmann, Acta Phys. Polon. B 40 (2009) 2139; PoS ICHEP2010 (2010) 150; F. Hautmann and H. Jung, Nucl. Phys. Proc. Suppl. 184 (2008) 64; arXiv:0812.3026; arXiv:0808.0873.

[35] S. Mert Aybat and T.C. Rogers, Phys. Rev. D83 (2011) 114042; P.J. Mulders and T.C. Rogers, Phys. Rev. D81 (2010) 094006; S. Mantry and F. Petriello, Phys. Rev. D84 (2011) 014030; Phys. Rev. D83 (2011) 053007; arXiv:1108.3609 [hep-ph]; T. Becher and M. Neubert, Eur. Phys. J. C 71 (2011) 1665 ; I.W. Stewart, F.J. Tackmann and W.J. Waalewijn, JHEP 1009 (2010) 005; A. Idilbi and I. Scimemi, Phys. Lett. B695 (2011) 463; arXiv:1012.4419 [hep-ph]; M. Garcia-Echevarria, A. Idilbi and I. Scimemi, arXiv:1111.4996 [hep-ph]; arXiv:1104.0686 
[hep-ph]; I. Cherednikov and N. Stefanis, arXiv:1104.0168 [hep-ph]; Phys. Rev. D80 (2009) 054008; Mod. Phys. Lett. A 24 (2009) 2913; F. Hautmann, Phys. Lett. B655 (2007) 26; arXiv:0708.1319; J.C. Collins and F. Hautmann, JHEP 0103 (2001) 016; Phys. Lett. B472 (2000) 129; hep-ph/0011381; hep-ph/0105098; hep-ph/0101006.

[36] F. Dominguez, A.H. Mueller, S. Munier and B.W. Xiao, arXiv:1108.1752 [hep-ph]; F. Dominguez, J.W. Qiu, B.W. Xiao and F. Yuan, arXiv:1109.6293 [hep-ph]; E. Avsar, arXiv:1108.1181 [hep-ph]; F. Dominguez, C. Marquet, B.W. Xiao and F. Yuan, Phys. Rev. D83 (2011) 105005; B.W. Xiao and F. Yuan, Phys. Rev. D82 (2010) 114009; Phys. Rev. Lett. 105 (2010) 062001; F. Dominguez, B.W. Xiao and F. Yuan, arXiv:1009.2141 [hep-ph]; F. Hautmann and D.E. Soper, Phys. Rev. D75 (2007) 074020; Phys. Rev. D63 (2000) 011501; F. Hautmann, Phys. Lett. B643 (2006) 171; hep-ph/0209320; hep-ph/0105082; F. Hautmann et al., hep-ph/9906284; hep-ph/9806298. 